\title{
Anatomy-shaped design of a fully-covered, biliary, self-expandable metal stent for treatment of benign distal biliary strictures
}

Authors

Institution
Jochen Weigt, Arne Kandulski, Peter Malfertheiner

Department of Gastroenterology, Hepatology and Infectious Diseases, Otto-von-Guericke University, Magdeburg, Germany
Bibliography

DOI http://dx.doi.org/

$10.1055 / \mathrm{s}-0034-1393583$

Published online: 27.11.2015

Endoscopy International Open

2016; 04: E79-E82

(c) Georg Thieme Verlag KG

Stuttgart $\cdot$ New York

E-ISSN 2196-9736

Corresponding author:

Jochen Weigt

Department of

Gastroenterology, Hepatology

and Infectious Diseases

Otto-von-Guericke University

Magdeburg, Germany

Phone: +493916713102

Fax: +493916713105

jochen.weigt@med.ovgu.de
Background and study aims: The treatment success of benign biliary strictures with fully covered metal stents (CSEMS) is altered by high stent dislocation rates. We aimed to evaluate a new stent design to prevent dislocation.

Patients and methods: Patients with benign biliary strictures were treated with a newly designed double-coned stent (dcSEMS). Mechanical analysis of the new stent was performed and it was compared with a cylindrical stent.

Results: A total of 13 dcCSEMS were implanted in 11 patients ( 2 female, 9 male, median age 47, range $33-71$ ). All patients had distal biliary strictures due to chronic pancreatitis. No stent migration occurred. In all but one patient the stents were removed. One patient refused stent extrac-

\section{Introduction}

$\nabla$

Benign biliary strictures are often refractory to simple treatment with placement of a single plastic stent or small-diameter dilatation therapy [1]. In such patients, placement of multiple plastic stents or of removable covered self-expandable metal stents (CSEMS) is appropriate [2-6]. Unfortunately the success rate of CSEMS treatment is reduced by migration of CSEMS, which occurs in up to $33 \%$ of cases, with the highest rates seen in patients with postsurgical stenosis after liver transplantation [7-10]. Some authors propose anchoring a CSEMS by use of a double pigtail plastic stent inside the CSEMS [9,11]. In a study by Park et al, the migration rate was lowered from $41.2 \%$ to $6.3 \%$ [9]. We propose that modification of the stent shape is more promising to prevent dislocation.

The goal of our research was to investigate the clinical performance of a newly designed CSEMS with special reference to the dislocation rate in a prospective, observational proof-of-concept study. We compared the mechanical properties tion and was lost to follow up. Stent occlusion occurred twice leading to cholangitis in both cases. The duration of stent treatment was 170 days (range 61-254). After extraction only one patient had early recurrent stricture and received the same stent again. Three stents showed minimal tissue granulation at the papilla. One stent presented ingrowth at the proximal end and was removed after implantation of a second fully covered stent.

Mechanical examination revealed significantly lower radial expansion force of the new stent as compared to the cylindrical stent.

Conclusions: The new stent design has a low rate of migration. Biomechanical properties may explain this effect.

of a new stent design with those of a standard cylindric stent.

\section{Patients and methods \\ $\nabla$ \\ Stent design}

The common bile duct is spindle shaped and narrows toward the papilla; narrowing also is common in cases of distal biliary stenosis. Commonly used biliary stents, however, typically are a uniform cylindrical shape and, therefore, do not fit into the bile duct, increasing the risk of dislocation. By using a double-coned stent design (dcCSEMS) that conforms to the natural shape of the common bile duct, migration forces on the stent can be equalized.

- Fig. 1 shows the newly designed stent, which has a maximum diameter of $12 \mathrm{~mm}$ at either end. At its narrowest, $15 \mathrm{~mm}$ away from the duodenal end, the diameter of the stent is $6 \mathrm{~mm}$. The device is available in $60-\mathrm{mm}$ and $80-\mathrm{mm}$ lengths. It is braided and consists of a single nitinol wire and is covered with a silicone membrane. 


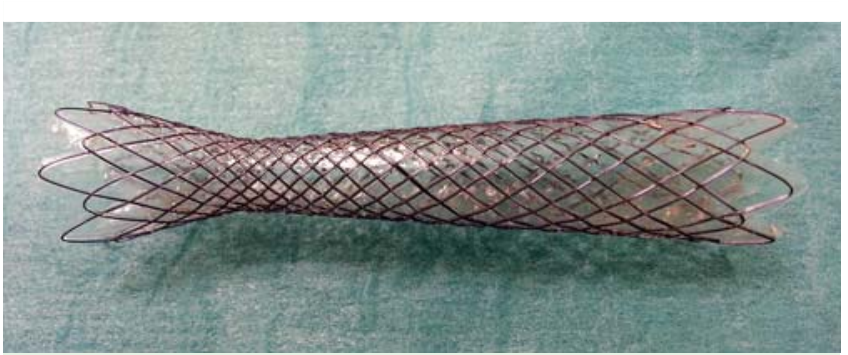

Fig. 1 Expanded stent with the duodenal end pointing to the left. The diameter at either end is $12 \mathrm{~mm}$ ( $36 \mathrm{~F}$ ) The notch is located $15 \mathrm{~mm}$ away from the duodenal end. The diameter at that position is $6 \mathrm{~mm}(18 \mathrm{~F})$. Stents were produced in total lengths of $60 \mathrm{~mm}$ and $80 \mathrm{~mm}$. The cover is made of silicone.

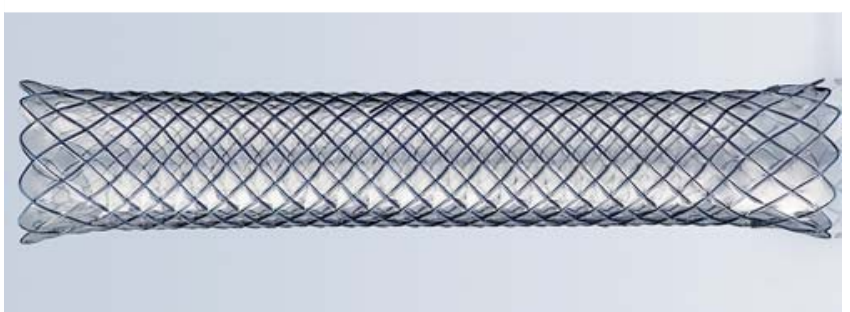

Fig.2 Image of the expanded fully-covered cylindrical stent (10-mm diameter, $80-\mathrm{mm}$ length) that was used for the mechanical analysis in comparison to the dcSEMS.

\section{Mechanical investigations}

We compared stents from the same manufacturer (Leufen Medical, Berlin, Germany). All stents were investigated on the same day in a water bath at $37^{\circ} \mathrm{C}$. Radial forces of the stents were measured during expansion and compression using an established measurement device (Zwick und Roell, Ulm, Germany). The first stent (Stent A) was an 80-mm dcCSEMS. The second stent (stent B) was a silicone-covered cylindric stent with a $10-\mathrm{mm}$ diameter and $80-\mathrm{mm}$ length ( $\bullet$ Fig.2). Measurements were performed at both ends of the stents, at $15 \mathrm{~mm}$ and $40 \mathrm{~mm}$. Each measurement was repeated seven times.

\section{Clinical study}

The clinical study was approved by the ethics committee of the University of Magdeburg (Ref. Nr.01/12) and was conducted as a prospective single-center observational study. The stents used in the study received CE mark before beginning of the study. Before inclusion all patients gave written informed consent.

A main inclusion criterion was the presence of untreated distal biliary stricture.

Previous treatment of a stenosis was defined as previous multistenting or SEMS placement and led to exclusion of patients. Patients with stenosis in the proximal third of the common bile duct were also not eligible. All patients had prior biliary sphincterotomy.

Patients were examined while in a prone position and under conscious sedation. The dcCSEMS (Leufen Medical, Aachen, Germany) was deployed on a 10-F application system over a 0.035 inch guide wire trough in the working channel of a side viewing endoscope (Olympus TJF-160VR, Olympus Europe, Hamburg, Germany or ED530XT8 Fujifilm Europe, Düsseldorf, Germany) by the same endoscopist (JW) ( $\odot$ Fig. 3a, $\odot$ Fig.3b, $\odot$ Fig. 3c). Follow up was performed regularly every 3 months or if complications occurred. Stents that showed no signs of ingrowth or dislocation were allowed to remain in place and were not exchanged. The main outcomes were stent dislocation rates, stent patency, and rates of complications.

\section{Results}

$\nabla$

\section{Mechanical Data}

The expansion forces of stent A (dcSEMS) at the duodenal end, the notch, the middle $(40 \mathrm{~mm})$ and the biliary end were $1.11 \mathrm{~N}$, $1.58 \mathrm{~N}, 1.10 \mathrm{~N}$ and $0.92 \mathrm{~N}$, respectively.

The maximal expansion forces of stent $\mathrm{B}$ at the ends $(15 \mathrm{~mm}$ and $40 \mathrm{~mm}$ ) were $2.99 \mathrm{~N}, 2.84 \mathrm{~N}$, and $2.78 \mathrm{~N}$, respectively.

In summary, the radial expansion forces of stent $\mathrm{A}$ were less than half that of the radial expansion forces of stent $B$. The exact measurements are given in $\bullet$ Fig. 4.
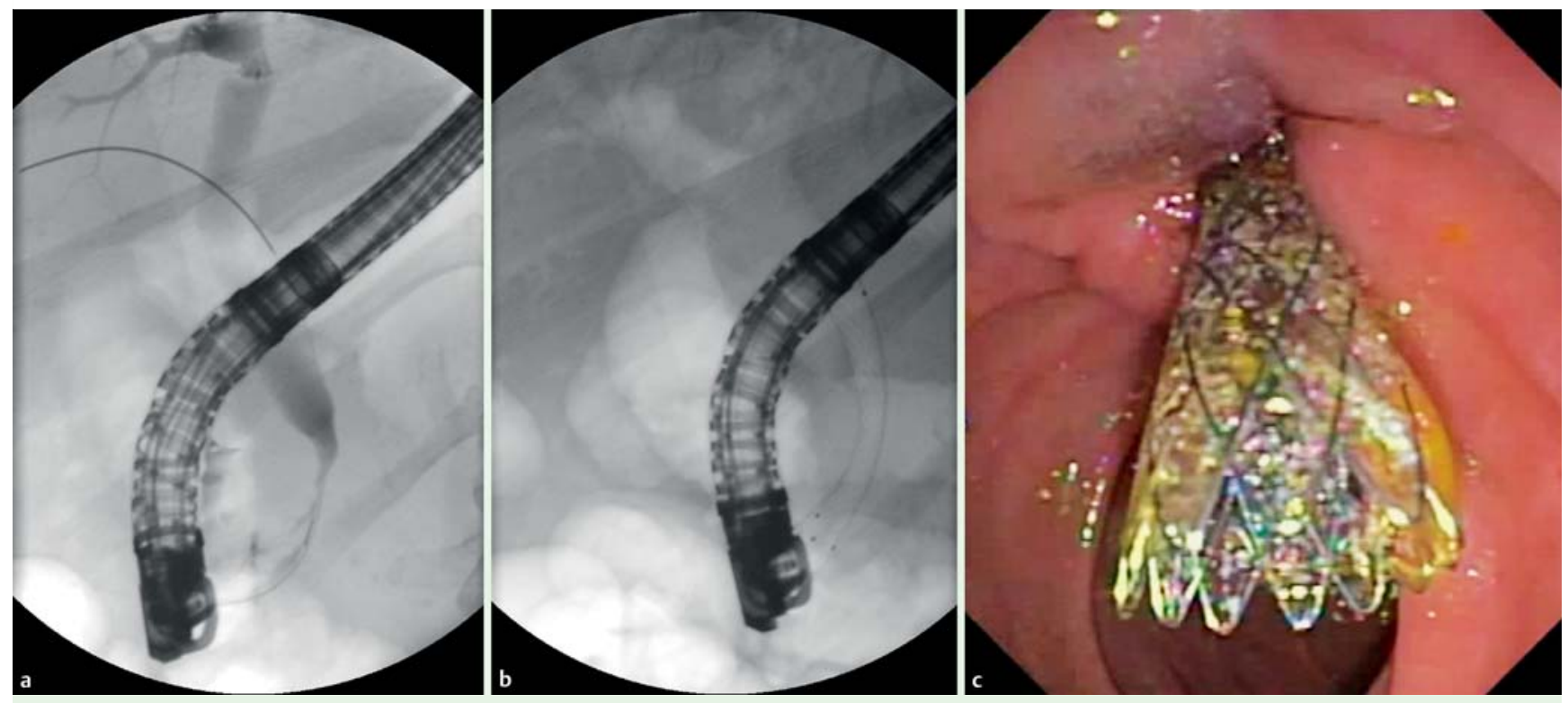

Fig. 3 a Distal biliary stenosis in a patient with chronic pancreatitis. b Fluoroscopic view of the stent immediately after deployment. c Endoscopic view of the stent after deployment. 

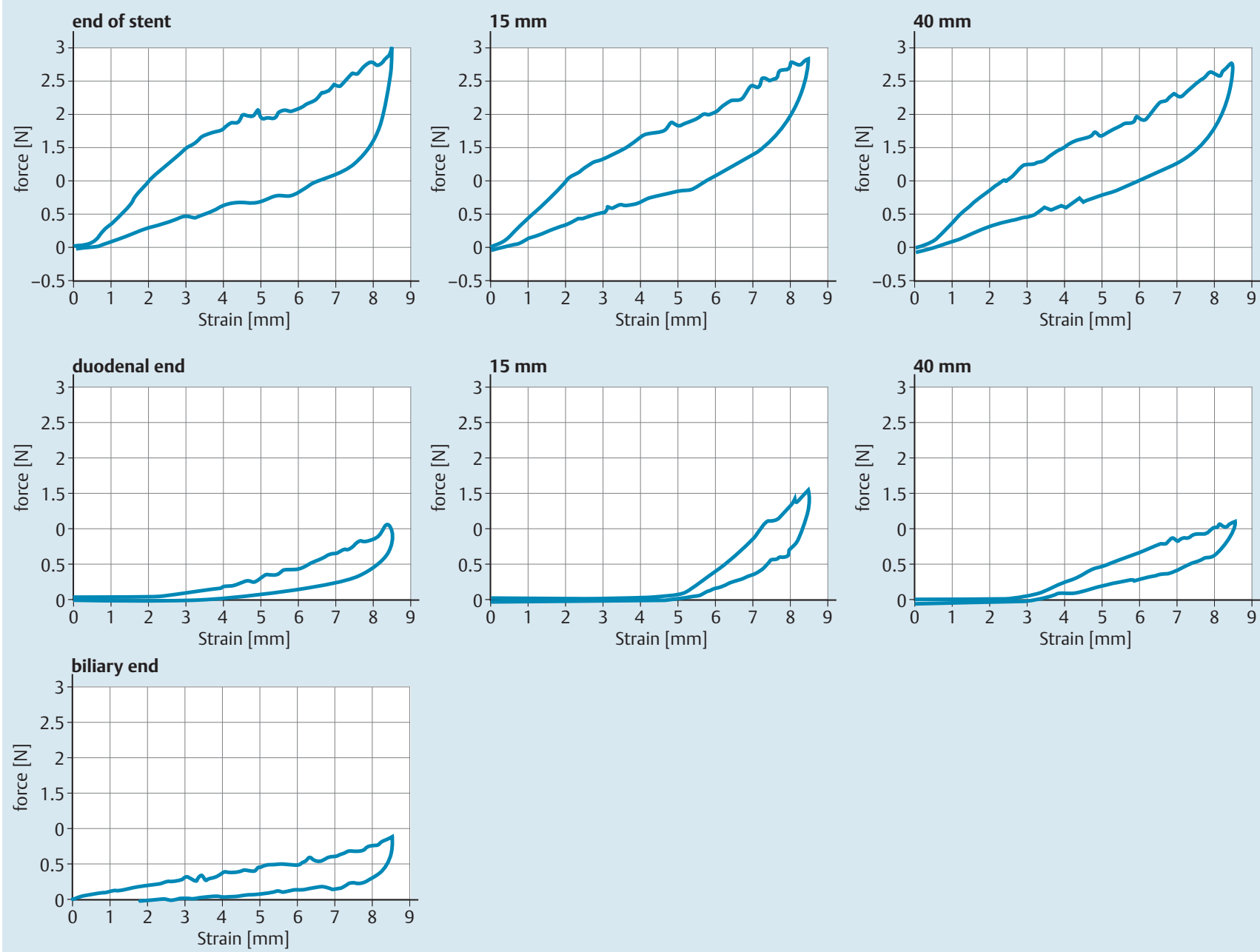

Fig. 4 Force strain plots expressing the radial forces of the stents during compression and extension. Upper panel: stent A, cylindrical. Lower panel: stent B double-coned stent.

\section{Clinical Data}

A total of 13 dcCSEMS were implanted in 11 patients (2 female, 9 male, median age 47 ; range $33-71$ ). All but one patient had distal biliary strictures due to chronic alcohol-induced pancreatitis. One patient had an anastomotic stricture after orthotopic liver transplantation that was located in the lower third of the common bile duct (CBD). Stent deployment was successful in all but one patient ( $91 \%$ ) in whom the stent did not cross the papilla and was replaced immediately. The mean duration of stent treatment was 170 days (range $61-254$. No stent migration occurred.

The stent was removed in all but one patient who refused stent extraction and was lost to follow up.In one patient the proximal end of the stent presented tissue ingrowth. With the help of intermittent implantation of a second covered stent (3-month period), extraction of the dsCSEMS was possible. Stent extraction was performed using a polypectomy snare or a biopsy forceps to grasp the duodenal end of the dcSEMS. The stents were removed through the working channel of the endoscope with the endoscope remaining in position Stent occlusion was observed three times leading to acute cholangitis in two cases, which were treated with stent extraction and antibiotic therapy. One patient died during observation because of newly diagnosed malignant disease. After extraction two patients had recurrent stricture, which occurred after 3 months in one patient with chronic pancreatitis and after 6 months in the patient with anastomotic stricture. In the patient with chronic pancreatitis, the same stent was placed again. In the other patient, the treatment was switched to dilatation and insertion of multiple 10-F plastic stents. The eight patients who had no evidence of recurrent stricture have been followed up for a mean of 616 days (range $242-868$ ) and needed no more treatment during this period.

\section{Discussion}

The new anatomy-adapted double-coned biliary covered stent has a low migration rate.

By reducing the stent diameter in some parts of the stenosis, the radial expansion force on the tissue is lower than with full-diameter stents. Depending on the position of the stent, the dilatation force inside the stenosis is lower than with larger-diameter stents. The target diameter of the stent in the area of the papilla is narrower because the natural shape of the CBD at that point is smaller than the rest of the duct.

In addition to the shape of the new stent, its softness (expressed in lower expansion forces) is an additional mechanism to reduce dislocation. We believe that it is not necessary to use a stent with a diameter up to $10 \mathrm{~mm}$ in all parts of the CBD to achiever treat- 
ment success and it may promote dislocation of cylindrical stents. That statement may appear to contradict the practice of using larger-diameter stents for treatment regimens with multistenting and other SEMS. In fact, dislocation of stents precludes treatment success and treatment failure is mainly caused by stent dislocation. Therefore, we suggest that prevention of stent dislocation can increase treatment success independent of stent diameter. However, the role of stent diameter should not be underemphasized.

Two recently published studies reinforce the usefulness of fullycovered SEMS for the treatment of benign biliary strictures and show high rates of stricture resolution (66\% and 76,3\%) [12,13]. However the study by Deviere et al [12] was designed to assess the safety of long-term indwelling and late extraction of SEMS and underlines the importance of prevention of stent dislocation. From a biomechanical point of view, the application of the new stent in its current shape is only useful for distal biliary stenosis. For the treatment of proximal stenosis, the shape of a stent should be configured accordingly.

A limitation to our study is the small number of patients included. The study was, however, designed as a pilot study to prove the concept of dcSEMS design. Because no fixed schedule for stent exchange was used in this study, the results regarding stent patency should be interpreted with caution.

In summary, our data demonstrate an effect of the stent shape on biomechanical behavior. Taking into consideration biomechanical and anatomical details may be important when designing stents for locations other than the gastrointestinal tract.

Competing interests: Dr. Weigt is an independent consultant for Leufen Medical, Berlin, Germany.

\section{Acknowledgements}

$\nabla$

The authors acknowledge their partnership with Christoph Leufen during the study and his support in the process for developing the stent.

\section{References}

1 Kahl S, Zimmermann S, Genz I et al. Risk factors for failure of endoscopic stenting of biliary strictures in chronic pancreatitis: a prospective follow-up study. Am J Gastroenterol 2003; 98: 2448-2453

2 Draganov $P$, Hoffman B, Marsh $W$ et al. Long-term outcome in patients with benign biliary strictures treated endoscopically with multiple stents. Gastrointest Endosc 2002; 55: 680-686

3 Dumonceau JM, Delhaye M, Tringali A et al. Endoscopic treatment of chronic pancreatitis: European Society of Gastrointestinal Endoscopy (ESGE) Clinical Guideline. Endoscopy 2012; 44: 784-800

4 Garcia-Cano J. Use of fully covered self-expanding metal stents in benign biliary diseases. World J Gastrointest Endosc 2012; 4: 142 - 147

5 Kao D, Zepeda-Gomez S, Tandon P et al. Managing the post-liver transplantation anastomotic biliary stricture: multiple plastic versus metal stents: a systematic review. Gastrointest Endosc 2013; 77: 679-691

6 Mahajan A, Ho H, Sauer B et al. Temporary placement of fully covered self-expandable metal stents in benign biliary strictures: midterm evaluation (with video). Gastrointest Endosc 2009; 70: 303 - 309

7 Moon JH, Choi HJ, Koo HC et al. Feasibility of placing a modified fully covered self-expandable metal stent above the papilla to minimize stent-induced bile duct injury in patients with refractory benign biliary strictures (with videos). Gastrointest Endosc 2012; 75: 1080 1085

8 Park do H, Lee SS, Lee TH et al. Anchoring flap versus flared end, fully covered self-expandable metal stents to prevent migration in patients with benign biliary strictures: a multicenter, prospective, comparative pilot study (with videos). Gastrointest Endosc 2011; 73: 64-70

9 Park JK, Moon JH, Choi $\mathrm{HJ}$ et al. Anchoring of a fully covered self-expandable metal stent with a $5 \mathrm{~F}$ double-pigtail plastic stent to prevent migration in the management of benign biliary strictures. Am J Gastroenterol 2011; 106: 1761 - 1765

10 Tarantino I, Mangiavillano B, Di Mitri R et al. Fully covered self-expandable metallic stents in benign biliary strictures: a multicenter study on efficacy and safety. Endoscopy 2012; 44: 923-927

11 Pausawasadi $N$, Soontornmanokul T, Rerknimitr R. Role of fully covered self-expandable metal stent for treatment of benign biliary strictures and bile leaks. Korean J Radiol 2012; 13: 0167-73

12 Deviere J, Nageshwar Reddy D, Puspok A et al. Successful management of benign biliary strictures with fully covered self-expanding metal stents. Gastroenterology 2014; 147: 385 - 395; quiz e315

13 Irani S, Baron TH, Akbar A et al. Endoscopic treatment of benign biliary strictures using covered self-expandable metal stents (CSEMS). Dig Dis Sci 2014; 59: $152-160$ 